\title{
Tail Pinch Facilitation of Self-Stimulation in the Rat-Dependence Upon Dopamine and Independence of Opiates
}

\author{
R. J. KATZ ${ }^{1}$, K. A. ROTH ${ }^{2}$ AND K. SCHMALTZ \\ Mental Health Research Institute, Department of Psychiatry, University of Michigan Medical Center, \\ Ann Arbor, MI 48109
}

Received 23 June 1979

\begin{abstract}
KATZ, R. J., K. A. ROTH AND K. SCHMALTZ. Tail pinch facilitation of self-stimulation in the rat-dependence upon dopamine, and independence of opiates. PHARMAC. BIOCHEM. BEHAV. 12(3) 389-391, 1980.-We have previously demonstrated that adult male Sprague-Dawley rats which are chronically maintained upon a schedule of intracranial reward (ICS) show elevated rates of response after a mild tail pinch. Both dopamine and opiates have been implicated in the mediation of other stress induced behavioral alterations, and may therefore also possibly be involved in the ICS effect. The present report replicated the initial finding of tail pinch induced facilitation of ICS, and further demonstrated that while opiate blockade failed to affect the ICS response dopaminergic blockade in fact inhibited it. These findings suggest neuropharmacological specificity for stress related behavioral change, and further implicate dopamine in stress responses.
\end{abstract}

Dopamine Endorphin Fluspirilene Naltrexone Self stimulation Stress Tail pinch

A VARIETY of behaviors including feeding, gnawing, sexual, maternal and aggressive behaviors may be elicited by mild non-traumatic tail pressure $[2,3,6,9]$. In a previous report [5] we noted that tail pinch may also increase responding for intracranial reward (ICS). While the pharmacological determinants of this last effect are unknown, if it is presumed to be related to other forms of self stimulation then involvement of catecholamines such as epinephrine [4] norepinephrine [8] and dopamine (DA), as well as possible involvement of endogenous opioid peptides (endorphins) might be predicted [8]. Tail pinch facilitation of ICS is in fact directly and significantly related to normal ICS [5], and therefore one or more of the above transmitters may mediate the tail pinch ICS syndrome.

The present report investigated the potential involvement of two of the above classes of transmitter, DA and endorphins, in the stress-arousal related facilitation of reward. These were considered likely initial choices for investigation on the basis of previous studies. On the one hand Antelman and colleagues have demonstrated clear DA dependency for many tail pinch related behaviors e.g. chewing and sexual behavior [2]. If tail pinch facilitation of ICS is related to the behaviors they have examined then DA may likewise be involved in its mediation. In a second design Akil and colleagues have demonstrated a correlated increase in opioid peptides with stress induced analgesia, as well as a partial reversal of this analgesia by the opiate blocker naloxone [1]. Since stress releases endorphins, and since they are involved in hedonic control [8] they may also potentially be involved in the tail pinch phenomenon. Both
DA and endorphins are therefore potential candidates for mediating the tail pinch phenomenon. To clarify the role or roles these transmitters may have we examined the effects of both DA and opiate blockade upon tail pinch induced self stimulation.

METHOD

\section{Subjects}

A total of 24 adult male Sprague-Dawley rats (Charles River Farms, Portage, MI) ranging from $300-500 \mathrm{~g}$ each were individually maintained with food (Teklad $4.0 \%$ fat rodent diet) and tap water continuously available. Lighting was automatically programmed on a $12 \mathrm{hr} / 12 \mathrm{hr}$ light/dark cycle (lights on $=0700-1900 \mathrm{hr}$ ) Normal $25 \times 18 \times 17 \mathrm{~cm}$ stainless steel cages were modified to house an overhead mounted stainless steel plate which allowed for continuous access to self stimulation as described below.

\section{Apparatus}

All testing was carried out in the subjects' home cages which were modified to allow for continuous self stimulation without external leads. Contact with a $14 \times 16 \mathrm{~cm}$ overhead mounted plate by a head mounted contact-brushing resulted in the delivery of a $0.3 \mathrm{sec}$ train of $60 \mathrm{cps}$ sinusoidal current of 20-60 $\mu \mathrm{A}$ current across a large $(50 \mathrm{k} \Omega)$ resistance and a zero-crossing relay to produce a constant current. Further details of the apparatus and current delivery have been presented in other publications (e.g., $[4,5])$.

${ }^{1}$ Correspondence to first author at above address.

${ }^{2}$ Kevin Roth is currently at the Pritzker Laboratory of Behavioral Neurochemistry, Stanford University, Palo Alto, CA. 


\section{Surgery}

Subjects were sterotactically implanted under $50 \mathrm{mg} / \mathrm{kg}$ of sodium pentobarital (Nembutal). A single monopolar $\mathbf{0 . 0 2 5}$ $\mathrm{cm}$ diameter nichrome wire insulated to tip was aimed at the medial forebrain bundle (MFB), with coordinates (in $\mathrm{mm}$ ) from $B r e g m a=-5.0$ (posterior), 1.0 (lateral) -8.0 (ventral) using a level skull. The electrode was secured to the skull and attached to the head mounted brushing with stainless steel screws and acrylic dental cement. At the close of all testing a subsample of six rats was randomly chosen and sacrificed for histological verification. Brains were sectioned at $40 \mu$ and stained with cresyl violet. All six rats' electrodes were within the MFB.

\section{Behavioral Procedure}

One week was initially allowed for recovery from surgery. The following two weeks were used for shaping and adjustment of current to maintain stable rates of response (final rates were $3600 \pm 1000$ responses $/ 24 \mathrm{hr}$; mean \pm SEM with $90 \%$ or more of responses made in the dark cycle). One additional week in which subjects were habituated to handling and experimental procedures preceded testing. Five sessions of handling and $2-4$ sessions of tail pinch preceded the experiments. All testing occurred during a period of normal behavioral quiescence (in all cases from 1400 to $1700 \mathrm{hr}$ ). Subjects received injections of either fluspirilene 1 or $2 \mathrm{mg} / \mathrm{kg}$ or Naltrexone 1 or $10 \mathrm{mg} / \mathrm{kg}$ administered $1 \mathrm{ml} / \mathrm{kg}$ intraperitoneally, $3 \mathrm{hr}$ or $1 \mathrm{hr}$ prior to experimental manipulation respectively. All drug or control injections utilized a $0.9 \%$ sodium chloride vehicle solution.

Two control procedures were utilized. The first was based upon a time sample of undisturbed responding during the test period. This did not involve actual cage removal. The second control procedure involved actual removal from the cage and mild handling. The experimental procedure involved subjecting the rats to a relatively mild tail pinch. A standard tongue forceps (i.e. rubber dam) closed to the first notch was applied $3-4 \mathrm{~cm}$ from the base of the tail for a 60 sec interval. This procedure produced pressure but no pain when tested on human fingers. Rats were returned to their cages after experimental or control procedures and their activity was recorded for the next $30 \mathrm{~min}$.

Subjects were tested on two to four occasions. In all cases repeated testing was carried out with sessions separated by a minimum of $72 \mathrm{hr}$, and only after normal response rate over $24 \mathrm{hr}$ had recovered to predrug rate $( \pm 10 \%)$. Order of administration of treatments was counterbalanced.

\section{Statistical Analysis}

Rates of response to tail pinch varied considerably across subjects. Because of this, and the possibility of unduely weighting scores of high responding animals all scores were percentage transformed prior to analysis. Response to saline-tail pinch was taken as $100 \%$, and results are expressed as \% mean and \% standard error. Analysis of effects across cells was by randomization test [7]. In all cases a minimum of six subjects (range 6-10) was used in the determination of cell means.

\section{TABLE 1}

TAIL PINCH FACILITATION OF SELF STIMULATION-EFFECTS OF DOPAMINE AND OPIATE BLOCKADE

Control rates (\% of experimental $\pm \%$ SE)

Control one (undisturbed):

Control two (handled):

$$
9 \pm 0^{*+\dagger}
$$

Tail pinch $(\%$ of experimental $\pm \%$ SE) Experimental group:

$100^{*}$

Fluspirilene rates ( $\%$ of experimental $\pm \% \mathrm{SE}$ ) $1 \mathrm{mg} / \mathrm{kg}$ :

$2 \mathrm{mg} / \mathrm{kg}$ :

$64 \pm 32$

$23 \pm 10^{\dagger}$

Naltrexone rates (\% of experimental $\pm \%$ SE)

$1 \mathrm{mg} / \mathrm{kg}$ :

$10 \mathrm{mg} / \mathrm{kg}$ :

$119 \pm 18$

$126 \pm 42$

*Untransformed scores for control $=0$; for handling $=87 \pm 39$; for experimental $=980 \pm 420 \mathrm{responses} / 30 \mathrm{~min}$.

+ Significantly different from experimental rate by randomization test for related samples; $p<0.05$ significance level.

\section{RESULTS}

Table 1 presents the main findings. It may be seen that little if any responding occurred when rats were undisturbed during the normal test interval. Vehicle injection and handling produced some albeit rather limited and statistically unreliable responding (column two). In comparison with either normal resting or vehicle injection, however, tail pinch in fact.elevated rates of responding. This elevation was not reduced by blocking opiate receptors, but was significantly reduced by dopamine blockers.

\section{DISCUSSION}

In the present report tail pinch facilitated self stimulation behavior. This was selectively reversed by DA blockade but not by opiate blockade. Opiate blockade has also proved ineffective in modifying other tail-pinch related phenomena. Wallach and colleagues found few effects of opiate or non opiate analgesics upon tail pinch related milk consumption except at behaviorally nonspecific doses. On the other hand dopamine blockers were effective in their model. These findings therefore both replicate earlier studies $[2,6]$ using self stimulation as a dependent variable and extend the hypothesis of Antelman and colleagues, i.e. that DA is a mediator of stress elicited behaviors [2].

It remains unclear from these or previous results whether a direct arousing effect or the aversive consequences of stimulation, or some combination of the above are chiefly responsible for the finding of behavioral facilitation. While we previously have argued that based upon behavioral observations aversion is one potential mediator [4] others have emphasized arousal per se $[2,6]$. If the DA system is considered an arousal system [2] then some support for the latter position may be obtained from these findings. 


\section{REFERENCES}

1. Akil, H., J. Madden IV, R. L. Patrick and J. D. Barchas. Stress induced increase in endogenous opiate peptides: Concurrent analgesia and its partial reversal by naloxone. In: Opiates and Endogenous Opiate Peptides, edited by H. W. Kosterlitz. Amsterdam: N. Holland Publishers, 1976, pp. 63-70.

2. Antelman, S. and A. R. Caggiula. Tails of stress related behavior: A neuropharmacological model. In: Animal Models in Psychiatry and Neurology, edited by I. Hanin and E. Usdin. Oxford: Pergamon Press, 1977, pp. 227-246.

3. Antelman, S., N. E. Rowland and A. E. Fisher. Stimulation Bound Ingestive Behavior: A view from the tail. Physiol. Behav. 17: $743-748,1976$.

4. Katz, R. J. and B. J. Carroll. Inhibition of phenylethanolamine-n-methyltransferase and brain stimulated reward. Psychopharmacology 57: 39-42, 1978.
5. Katz, R. J. and K. Roth. Tail pinch induced stress-arousal facilitates brain stimulation reward. Physiol. Behav. 22: 193-194, 1979.

6. Koob, G. F., P. Fray and S. D. Iversen. Tail pinch stimulation: sufficient motivation for learning. Science 194: 637-639, 1976.

7. Siegel, S. Non Parametric Statistics for the Behavioral Sciences. New York: McGraw Hill, 1956.

8. Stein, L. Reward transmitters: Catecholamines and opioid peptides. In: Psychopharmacology: A Generation of Progress, edited by M. A. Lipton, A. Dimasceo and K. F. Killam. New York: Raven Press, pp. 569-582.

9. Wallach, M. D., M. Dawber, M. McMahon and C. Rogers. A new anorexigen assay: stress induced hyperphagia in rats. Pharmac. Biochem. Behav. 6: 529-531, 1977. 